\title{
Spontaneous Symmetry Breaking in a Non-Conserving Two-Species Driven Model
}

\author{
E. Levine $\dagger$ and R. D. Willmann $\ddagger$ \\ $\dagger$ Department of Physics of Complex Systems, Weizmann Institute of Science, \\ Rehovot, Israel 76100. \\ $\ddagger$ Institut für Festkörperforschung, Forschungszentrum Jülich, 52425 Jülich, Germany
}

\begin{abstract}
A two species particle model on an open chain with dynamics which is non-conserving in the bulk is introduced. The dynamical rules which define the model obey a symmetry between the two species. The model exhibits a rich behavior which includes spontaneous symmetry breaking and localized shocks. The phase diagram in several regions of parameter space is calculated within mean-field approximation, and compared with Monte-Carlo simulations. In the limit where fluctuations in the number of particles in the system are taken to zero, an exact solution is obtained. We present and analyze a physical picture which serves to explain the different phases of the model.

PACS numbers: 05.70.Ln, 64.60.Ht, 02.50.Ga, 47.70.-n
\end{abstract}

\section{Introduction}

One dimensional driven diffusive systems have been shown in the last decade to demonstrate a variety of non-trivial types of behavior. In contrast to equilibrium systems with local interactions, driven systems were shown to exhibit boundary induced phase transitions, phase separation and spontaneous symmetry breaking even when the dynamics is local [1, 2, 3, 4, 5]. The latter phenomenon was first demonstrated for a driven two-species model with open boundary conditions, which became known as the 'Bridge model' [6, 2]. This model is defined on an open lattice, where two species of particles, which can be thought of as carrying opposite 'charges', are moving in opposite directions. Although the dynamical rules which define the model are symmetric under charge exchange and left-right reflection, a mean-field approach and Monte Carlo simulations showed the existence of two phases in which this symmetry is broken. This result was subsequently supported by an exact solution for the limit of small extraction rates at the boundaries [7].

Until recently, studies of one dimensional driven systems focused on conserving bulk dynamics. Non-conserving dynamics was only considered at certain sites, namely defect sites or boundaries. Recently, the existence of phase transitions in driven systems 
without bulk particle conservation was demonstrated, both for finite non-conserving rates [10] and for rates which scale to zero with the system size [11, 13, 12 .

In this work we study a variant of the Bridge model, in which non-conserving dynamics is introduced in the bulk of the system. All dynamical rates, both conserving and non-conserving, respect the CP-symmetry between the two species of particles. By its nature, this dynamics acts to balance the densities of the two species. If the nonconserving transitions occur at finite rates, spontaneous symmetry breaking does not occur. However, we find that when these rates are inversely proportional to the system size, spontaneous symmetry breaking appears. This model is related to the singlespecies non-conserving asymmetric exclusion process which was introduced and studied recently [11,13, 12. As in that model, the two-species model exhibits new phases with localized shocks. Transitions in the phase diagram can be understood by considering the position of the localized shocks. Furthermore, the phenomenon of induced localized shocks is observed, as predicted in [14].

The paper is organized as follows. The next section contains the definition of the model. In section 3 we calculate the phase diagram within mean-field approximation, for some cases where the bulk dynamics of the two species are decoupled. Different limits of the model are discussed, and in particular the phase diagram of the Bridge model is recovered as the non-conserving rates are reduced to zero. Next, we present in section 4 results of Monte-Carlo simulations, and compare them with the predictions of mean-field analysis. The phenomenon of induced shocks is addressed in section 5. In section 6] we present an exact solution for the case where fluctuations in the number of particles in the system are taken to zero. A physical picture is introduced and analyzed in section 7. We conclude and summarize in section 8 .

\section{Model Definition}

The model considered in this paper is defined on a one-dimensional lattice of size $N$. Each lattice site can either be occupied by a positive $(+)$ particle, occupied by a negative $(-)$ particle, or vacant $(0)$. The system evolves through three types of stochastic rates: In the bulk of the system particles move on the lattice according to the rates

$$
+0 \stackrel{1}{\rightarrow} 0+\quad+-\stackrel{q}{\rightarrow}-+\quad 0-\stackrel{1}{\rightarrow}-0 .
$$

In addition, each site in the bulk of the system, $1<i<N$, can change its state with rates

$$
+\frac{\omega_{X}}{\rightleftharpoons}-\quad+\frac{\omega_{D}}{\omega_{X}} 0 \quad-\frac{\omega_{D}}{\rightleftharpoons} 0,
$$

corresponding to charge exchange, desorption of a particle from the lattice, and adsorption of a particle at an empty site. At the boundaries particles may be introduced and removed. At the left boundary, site $i=1$, positive particles are introduced and negative particles are removed with rates

$$
0 \stackrel{\alpha}{\rightarrow}+\quad-\stackrel{\beta}{\rightarrow} 0 \quad(i=1)
$$


while at the right boundary, $i=N$, negative particles are introduced and positive particles are removed with rates

$$
0 \stackrel{\alpha}{\rightarrow}-\quad+\stackrel{\beta}{\rightarrow} 0 \quad(i=N) .
$$

Note that all dynamical rules, conserving and non-conserving, are CP-symmetric, namely symmetric under the exchange of positive-negative charges and left-right directions. Generalizations of this model to the case were both types of particles can move in both directions, and when the dynamical rules break the CP symmetry, will be considered elsewhere.

Considering the bulk non-conserving rates, one distinguishes three possible scenarios [13. If the rates are finite, in the thermodynamic limit the bulk densities are dominated by the non conserving kinetics. Otherwise, if the rates decay to zero faster than $1 / N$, the bulk non-conservation should have no effect, and the properties of the system in the thermodynamic limit are identical to those with bulk conservation. The third case, which we consider here, is the one in which the non-conserving rates scale down linearly with the system size. It is useful to introduce the notation $\omega_{A}=\Omega / N$, $\omega_{X}=\Omega u_{X} / N, \omega_{D}=\Omega u_{D} / N$.

Without the non-conserving dynamics in the bulk of the system, Eq. (2), the model is identical to the Bridge model introduced in [6] and further studied in [7, 9, 8. Let us summarize first the known results for this model. At $q=1$ mean-field analysis predicts the following phase diagram [6]. Two phases which obey the CP-symmetry of the model are identified: a maximal-current phase, where the bulk density of each species equals $\frac{1}{2}$, and a low-density phase, where the bulk densities of both species are identical, both smaller than $\frac{1}{2}$. In addition, the model exhibits two phases in which the symmetries are broken. In one phase, termed the Low-High phase, one of the species, spontaneously chosen, sustains a bulk density larger than $\frac{1}{2}$, while the bulk density of the other is smaller than $\frac{1}{2}$. In the other phase both species sustain low albeit non-equal densities. This phase spans a very small area of the mean-field phase diagram, and is very difficult to recover in Monte-Carlo simulations. Consequently, the existence of this low-density asymmetric phase under noisy dynamics has been the subject of some debate [9, 8 .

The model considered here can be thought of as two interacting single-species totally asymmetric exclusion processes with bulk non-conservation (NC-TASEP). The NCTASEP is an extension of the well-known totally asymmetric exclusion process (TASEP) 15. with bulk absorption and desorption dynamics. Using mean-field calculations [12], which were argued to be exact [13, the phase diagram of this model was obtained. In addition to the three phases of the TASEP, namely the maximal-current phase, the high-density phase and the low-density phase, it was found that the NC-TASEP may also exhibit four additional phases. The most interesting one is a Shock phase which consists of a localized shock in the bulk of the system, separating a low-density region to its left from a high-density region to its right. In the bulk-conserving TASEP shocks appear only on the boundary line between the high-density and low-density phases. On that line a delocalized shock appears in the system. As the position of the shock is 
equally probable at any site in the system, the average profile on the transition line is linear. In contrast, the NC-TASEP exhibits a distinct phase in which a localized shock, whose position is selected by the dynamics, is present. The existence of this phase plays a main role in our analysis of the two-species model.

The maximal-current phase of the TASEP appears in the NC-TASEP only when the two non-conserving rates, namely particle absorption and desorption, are equal. In this case there exist three more phases. These include a Low-Max phase, in which the density increases linearly from a boundary density $<\frac{1}{2}$ towards density $\frac{1}{2}$, where it remains constant for the rest of the system ; a Max-High phase, in which the density rises linearly from a constant profile of density $\frac{1}{2}$ to a boundary density $>\frac{1}{2}$; and a Low-Max-High phase, in which the density rises linearly from a left-boundary density $<\frac{1}{2}$ towards $\frac{1}{2}$, where it remains constant up to a point where it climbs linearly again towards a right-boundary density $>\frac{1}{2}$.

\section{Mean Field Theory}

In this section we study the mean-field equations of our model in the thermodynamic limit $N \rightarrow \infty$. We introduce the occupation variables $\tau_{i}$ and $\theta_{i}$, such that $\tau_{i}=1\left(\theta_{i}=1\right)$ if site $i$ is occupied by a positive (negative) particle, and 0 otherwise. The densities of the positive and negative particles are then defined by

$$
p_{i}=\left\langle\tau_{i}\right\rangle \quad m_{i}=\left\langle\theta_{i}\right\rangle
$$

where angular brackets denote averaging over realizations.

The time evolution of the particle densities is governed by

$$
\begin{aligned}
& \frac{d p_{i}}{d t}=j_{i-1}^{+}-j_{i}^{+}+S_{i}^{+} \\
& \frac{d m_{i}}{d t}=j_{i+1}^{-}-j_{i}^{-}+S_{i}^{-},
\end{aligned}
$$

where the currents are given by

$$
\begin{aligned}
& j_{i}^{+}=\left\langle\tau_{i}\left(1-\tau_{i+1}-(1-q) \theta_{i+1}\right)\right\rangle \\
& j_{i}^{-}=\left\langle\theta_{i}\left(1-\theta_{i-1}-(1-q) \tau_{i-1}\right)\right\rangle,
\end{aligned}
$$

and the source terms are

$$
\begin{aligned}
& S_{i}^{+}=\omega_{A}\left(1-p_{i}-m_{i}\right)-\omega_{D} p_{i}+\omega_{X}\left(m_{i}-p_{i}\right) \\
& S_{i}^{-}=\omega_{A}\left(1-m_{i}-p_{i}\right)-\omega_{D} m_{i}+\omega_{X}\left(p_{i}-m_{i}\right) .
\end{aligned}
$$

At the boundaries the source terms vanish, and the currents are given by

$$
\begin{aligned}
& j_{0}^{+}=\alpha\left(1-p_{1}-m_{1}\right) \\
& j_{N}^{+}=\beta p_{N} \\
& j_{1}^{-}=\beta m_{1} \\
& j_{N+1}^{-}=\alpha\left(1-p_{N}-m_{N}\right) .
\end{aligned}
$$


The mean-field theory for this model is defined by replacing two-point correlation functions with products of one-point averages. Within this approximation, the currents become

$$
\begin{aligned}
& j_{i}^{+}=p_{i}\left(1-p_{i+1}-(1-q) m_{i+1}\right) \\
& j_{i}^{-}=m_{i}\left(1-m_{i-1}-(1-q) p_{i-1}\right) .
\end{aligned}
$$

In the steady-state all time derivatives vanish, and one has

$$
j_{i}^{+}=j_{i-1}^{+}+S_{i}^{+} \quad j_{i}^{-}=j_{i+1}^{+}+S_{i}^{+} .
$$

Defining $\mathcal{J}_{i}^{+}=j_{i}^{+}-\sum_{k=0}^{i} S_{k}^{+}, \mathcal{J}_{i}^{-}=j_{i}^{-}-\sum_{k=i}^{N} S_{k}^{-}$, one notices that in fact $\mathcal{J}_{i}^{+} \equiv \mathcal{J}^{+}$ and $\mathcal{J}_{i}^{-} \equiv \mathcal{J}^{-}$are conserved throughout the lattice, and $\mathcal{J}^{+}=j_{0}^{+}, \mathcal{J}^{-}=j_{N+1}^{-}$.

\subsection{Solution of the mean-field equations in the bulk-decoupled case}

The case $q=1, u_{X}=1$ is special, as in this case the bulk equations (6) with the mean-field currents (10) are decoupled. The currents and source terms in this case are just

$$
\begin{aligned}
j_{i}^{+} & =p_{i}\left(1-p_{i+1}\right) & S_{i}^{+} & =\frac{\Omega}{N}\left[1-\left(2+u_{D}\right) p_{i}\right] \\
j_{i}^{-} & =m_{i}\left(1-m_{i-1}\right) & S_{i}^{-} & =\frac{\Omega}{N}\left[1-\left(2+u_{D}\right) m_{i}\right] .
\end{aligned}
$$

In the bulk of the system, the hopping rates for say a positive particle do not distinguish between a negative particle and a vacancy. Also, the fact that attachment of a positive particle is limited by the presence of negative ones, is exactly compensated by the charge exchange rate. The coupling between the two species is limited in this case only to the boundaries. Following [6], one readily notices that upon the definition

$$
\begin{aligned}
& \alpha^{+}=\frac{\alpha\left(1-p_{1}-m_{1}\right)}{1-p_{1}}=\frac{\mathcal{J}^{+}}{\frac{\mathcal{J}^{+}}{\alpha}+\frac{\mathcal{J}^{-}+\mathcal{S}^{-}}{\beta}}=\frac{j_{0}^{+}}{\frac{j_{0}^{+}}{\alpha}+\frac{j_{0}^{-}}{\beta}} \\
& \alpha^{-}=\frac{\alpha\left(1-p_{N}-m_{N}\right)}{1-m_{N}}=\frac{\mathcal{J}^{-}}{\frac{\mathcal{J}^{-}}{\alpha}+\frac{\mathcal{J}^{+}+\mathcal{S}^{+}}{\beta}}=\frac{j_{0}^{-}}{\frac{j_{N}^{-}}{\alpha}+\frac{j_{N}^{+}}{\beta}},
\end{aligned}
$$

with $S^{ \pm}=\sum_{i=1}^{N} S_{i}^{ \pm}$, the problem is reduced to two single-species NC-TASEP. One process corresponds to the positive particles with injection rate $\alpha^{+}$at the left boundary and ejection rate $\beta$ at the right, and the other to the negative particles with injection rate $\alpha^{-}$at the right boundary and ejection rate $\beta$ at the left. The two processes may or may not share the same phase. The latter case corresponds to a phase of the twospecies system, where the symmetry between the two species is broken. In the other case, it may be that the average densities of the two species are not equal, although the two lie in the same phase of the corresponding NC-TASEP. A trivial restriction on the possible phases in the model is $p_{i}+m_{i} \leq 1$ at all sites. This immediately excludes several possibilities, such as ones which mix the high-density phase of one species with anything but the low-density of the other.

In this section we explore the possible phases in the bulk decoupled case. Symmetric phases are presented first, followed by asymmetric phases. In the symmetric phases, 

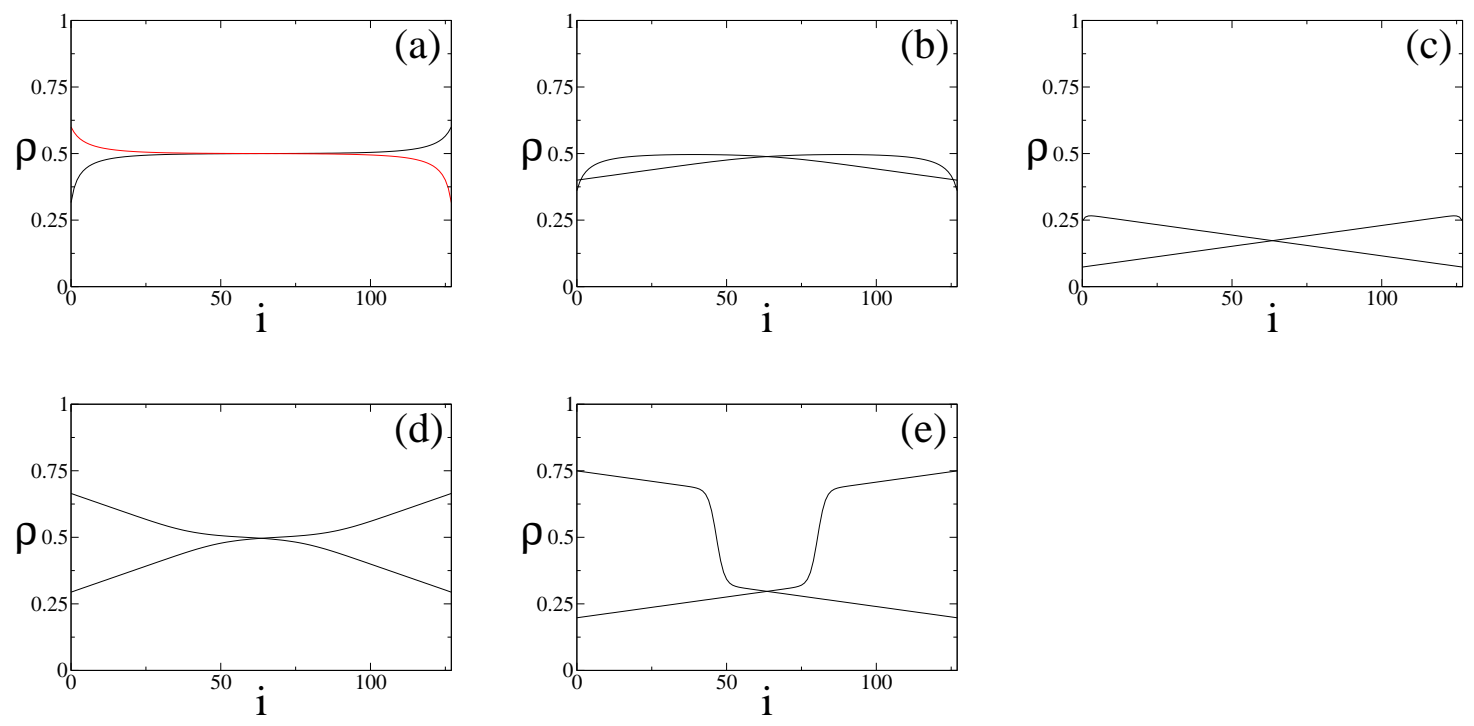

Figure 1. Density profiles of the symmetric phases, as obtained by integrating the mean-field equations for a system of size $N=128$. (a) Max Phase, $\alpha=3.0, \beta=0.8$, $\Omega=0.2, q=1$. (b) Low-Max Phase, $\alpha=1.0, \beta=0.7, \Omega=0.2, q=1$. (c) Low Phase, $\alpha=0.1, \beta=0.8, \Omega=0.2, q=1$. (d) Low-Max-High Phase, $\alpha=5.0, \beta=1 / 3$, $\Omega=0.5, q=1$. (e) Shock Phase, $\alpha=3.0, \beta=0.25, \Omega=0.2, q=1$.

$\alpha^{+}=\alpha^{-}$, so for these phases only $\alpha^{+}$is quoted in the following. For the asymmetric phases it is always assumed, with no loss of generality, that the positive particles are in the majority. When describing density profiles we always take a language in which the lattice is rescaled to the segment $[0,1]$. The emerging phase diagram is discussed in the following subsection.

3.1.1. Maximal-current symmetric phase. In this phase the bulk density of both species is $\frac{1}{2}$, and the boundary currents are given by

$$
j_{0}^{+}=j_{0}^{-}=\frac{1}{4}
$$

The conditions for this phase to exist are

$$
\alpha^{+}>\frac{1}{2} \quad \beta>\frac{1}{2} .
$$

Typical density profiles of the two species in this phase, as obtained from integrating the mean-field equations, are shown in figure 1 (a).

3.1.2. Low - Max symmetric phase. The density profile in this phase is composed of a low density part where the density increases linearly with slope $\Omega$ on the rescaled lattice, as well as a part with constant density $\frac{1}{2}$. The boundary currents are

$$
j_{0}^{+}=\alpha^{+}\left(1-\alpha^{+}\right) \quad j_{0}^{-}=\frac{1}{4} .
$$


The conditions for the existence of this phase are

$$
\alpha^{+}<\frac{1}{2} \quad \alpha^{+}>\frac{1}{2}-\Omega \quad \beta<\frac{1}{2} .
$$

The density profiles shown in figure 1 (b) for a finite system furthermore exhibits a boundary layer, which does not scale with the system size.

3.1.3. Low density symmetric phase. In this phase both densities remain below $\frac{1}{2}$, increasing throughout the system with constant slope $\Omega$ (figure 1(c)). The boundary currents are given by

$$
j_{0}^{+}=\alpha^{+}\left(1-\alpha^{+}\right) \quad j_{0}^{-}=\left(\alpha^{+}+\Omega\right)\left(1-\alpha^{+}-\Omega\right) .
$$

Necessary conditions for the existence of this phase are

$$
\begin{aligned}
& \alpha^{+}<\beta-\Omega \text { for } \beta<\frac{1}{2} \\
& \alpha^{+}<\frac{1}{2}-\Omega \text { for } \beta \geq \frac{1}{2} .
\end{aligned}
$$

Inserting the boundary currents (18) into (13) yields a quadratic equation for $\alpha^{+}$. Using (19) one readily identifies the relevant solution.

3.1.4. Low - Max - High symmetric phase. The density profiles in this phase are a mixture of three different pieces - a linear profile of low densities and constant slope $\Omega$, followed by a flat density profile at density $\frac{1}{2}$, and a linear profile of high-densities of the same slope (figure 1(d)). The boundary currents are now

$$
j_{0}^{+}=\alpha^{+}\left(1-\alpha^{+}\right) \quad j_{0}^{-}=\beta(1-\beta) .
$$

This phase region is defined by the conditions

$$
\alpha^{+}>\frac{1}{2}-\Omega \quad \beta<\frac{1}{2} .
$$

Again, one solves the equation for $\alpha^{+}$given by (13) and (20), and uses (21) to identify the relevant solution. For a finite system (as seen in figure 1 (d)) the transition between the three parts is not sharp. It only becomes so on the rescaled lattice as $N \rightarrow \infty$.

3.1.5. Shock symmetric phase. This phase is characterized by a localized shock, separating a low-density region from a high-density one, both of linear profile with slope $\Omega$. The boundary currents are given by

$$
j_{0}^{+}=\alpha^{+}\left(1-\alpha^{+}\right) \quad j_{0}^{-}=\beta(1-\beta) .
$$

Notice that $\alpha^{+}$in this phase is identical to the one of the Low-Max-High phase. The Shock symmetric phase is defined by the conditions

$$
\beta-\Omega<\alpha^{+}<\frac{1}{2}-\Omega .
$$

The position of the shock $x_{s}$ is given by

$$
x_{s}=\frac{\beta-\alpha^{+}}{2 \Omega}+\frac{1}{2}=\frac{2 \beta-(1+\alpha)+\sqrt{(1+\alpha)^{2}-4 \alpha \beta}}{4 \Omega}+\frac{1}{2} .
$$



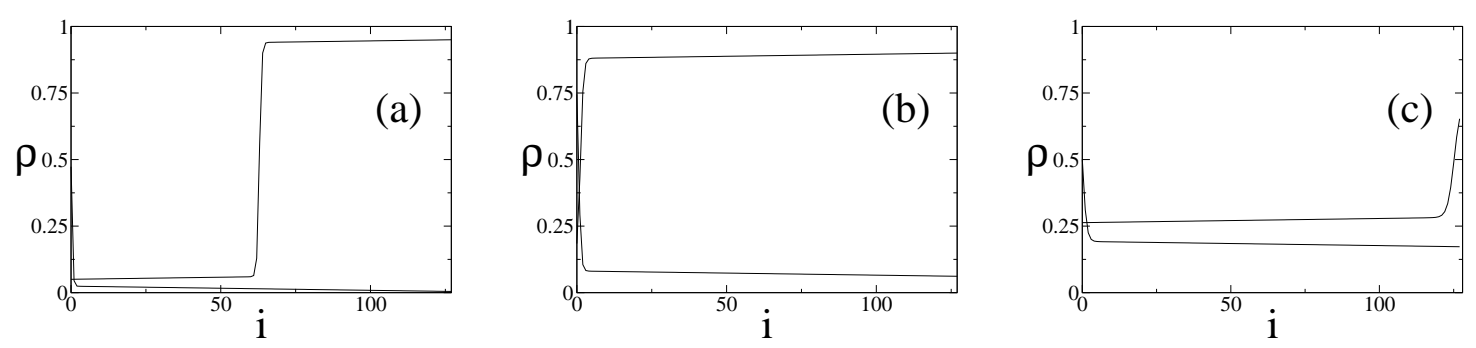

Figure 2. Density profiles of the asymmetric phases, as obtained by integrating the mean-field equations for a system of size $N=128$. (a) Shock-Low asymmetric Phase, $\alpha=0.1, \beta=0.05, \Omega=0.02, q=1$. (b) High-Low asymmetric Phase, $\alpha=1.5, \beta=0.1$, $\Omega=0.02, q=1$. (c) Low asymmetric Phase, $\alpha=0.82, \beta=0.31, \Omega=0.02, q=1$.

In contrast to the thermodynamical limit, the shocks in a finite system such as in figure 1) (e) are not sharp.

3.1.6. Shock - Low asymmetric phase In this phase the majority species exhibits a localized shock, while the minority species is in the Low phase throughout the system (figure 2 (a)). The boundary currents for the two species are

$$
\begin{array}{ll}
j_{0}^{+}=\alpha^{+}\left(1-\alpha^{+}\right) & j_{0}^{-}=\left(\alpha^{-}+\Omega\right)\left(1-\alpha^{-}-\Omega\right) \\
j_{N}^{+}=\beta(1-\beta) & j_{N}^{-}=\alpha^{-}\left(1-\alpha^{-}\right) .
\end{array}
$$

The conditions for the existence of this phase are given by

$$
\alpha^{+}>\beta-\Omega \quad \alpha^{+}<\beta+\Omega \quad \beta<\frac{1}{2} .
$$

The equation for $\alpha^{-}$does not involve $\alpha^{+}$, and can be solved as in previous phases. Plugging this solution into the equation for $\alpha^{+}$one can solve the equation, and identify the only solution which obeys (26).

3.1.7. High - Low asymmetric phase This phase is analogous to the strong asymmetric phase of the Bridge model. In this phase the majority sustains a high density in the bulk of the system, while the minority density is low. Here, however, the density profiles are not constant, but rather of opposite slopes $\pm \Omega$ (figure 2 (b)). The boundary currents for the two species are

$$
\begin{array}{ll}
j_{0}^{+}=(\beta+\Omega)(1-\beta-\Omega) & j_{0}^{-}=\left(\alpha^{-}+\Omega\right)\left(1-\alpha^{-}-\Omega\right) \\
j_{N}^{+}=\beta(1-\beta) & j_{N}^{-}=\alpha^{-}\left(1-\alpha^{-}\right) .
\end{array}
$$

The conditions for the existence of this phase are

$$
\alpha^{+}>\beta+\Omega \quad \beta+\Omega<\frac{1}{2} .
$$

Expressions for $\alpha^{ \pm}$are obtained from (27), (28) just as in the Shock-Low phase. 
3.1.8. Low asymmetric phase In this phase both particle species maintain a low density profile with constant slope $\Omega$. Still, the phase is asymmetric as the boundary densities of the two phases are different. An analogous phase is also observed on the mean field level in the Bridge model. The boundary currents are given by

$$
\begin{array}{ll}
j_{0}^{+}=\alpha^{+}\left(1-\alpha^{+}\right) & j_{0}^{-}=\left(\alpha^{-}+\Omega\right)\left(1-\alpha^{-}-\Omega\right), \\
j_{0}^{+}=\alpha^{-}\left(1-\alpha^{-}\right) & j_{0}^{+}=\left(\alpha^{+}+\Omega\right)\left(1-\alpha^{+}-\Omega\right) .
\end{array}
$$

Plugging the currents (29) into (13) gives

$$
\begin{aligned}
& \alpha^{+}=1-\frac{\alpha^{+}\left(1-\alpha^{+}\right)}{\alpha}-\frac{\left(\alpha^{-}+\Omega\right)\left(1-\alpha^{-}-\Omega\right)}{\beta} \\
& \alpha^{-}=1-\frac{\alpha^{-}\left(1-\alpha^{-}\right)}{\alpha}-\frac{\left(\alpha^{+}+\Omega\right)\left(1-\alpha^{+}-\Omega\right)}{\beta}
\end{aligned}
$$

Let $D=\alpha^{+}-\alpha^{-}$and $S=\alpha^{+}+\alpha^{-}$. Subtracting the equations in (30) yields

$$
D=D\left((1-S) \frac{\alpha-\beta}{\alpha \beta}-\frac{2 \Omega}{\beta}\right) \text {. }
$$

In the asymmetric phase, $D \neq 0$, thus an expression for $S$ is obtained. Summing the equations in (30) and using this result yields $D$ as a function of $\alpha$ and $\beta$. The effective boundary rates are obtained as $\alpha^{+}=\frac{1}{2}(S+D)$ and $\alpha^{-}=\frac{1}{2}(S-D)$. Necessary conditions for the existence of this phase are

$$
\begin{aligned}
& \alpha^{+}<\beta-\Omega \text { and } \beta<\frac{1}{2} \\
& \alpha^{+}<\frac{1}{2}-\Omega \text { and } \beta \geq \frac{1}{2} \\
& D>0 .
\end{aligned}
$$

A typical profile for this phase is shown in figure 2 (c).

\subsection{Phase Diagram}

In the previous section we have listed all phases which are found to exist in this model, and derived the phase boundaries in which they reside in parameter space. We now turn to describe the emerging phase diagram. First note that the full parameter space is covered by the four symmetric phases. In fact, all asymmetric phases reside in regions of phase space where the low-density symmetric state is also stationary. Which of the solutions is realized is a matter of stability, as will be discussed shortly. Except for the boundary of the Maximal-current phase, the phase boundaries of the symmetric phases share a common point $Q$ in the $(\alpha, \beta)$ plane, given by

$$
Q=\left(\frac{1-4 \Omega^{2}}{4 \Omega}, \frac{1}{2}\right) .
$$

The intersection point of the phase boundary between the Low and the Shock symmetric phases meets the $\beta$ axis at the point

$$
R=(0, \Omega) \text {. }
$$



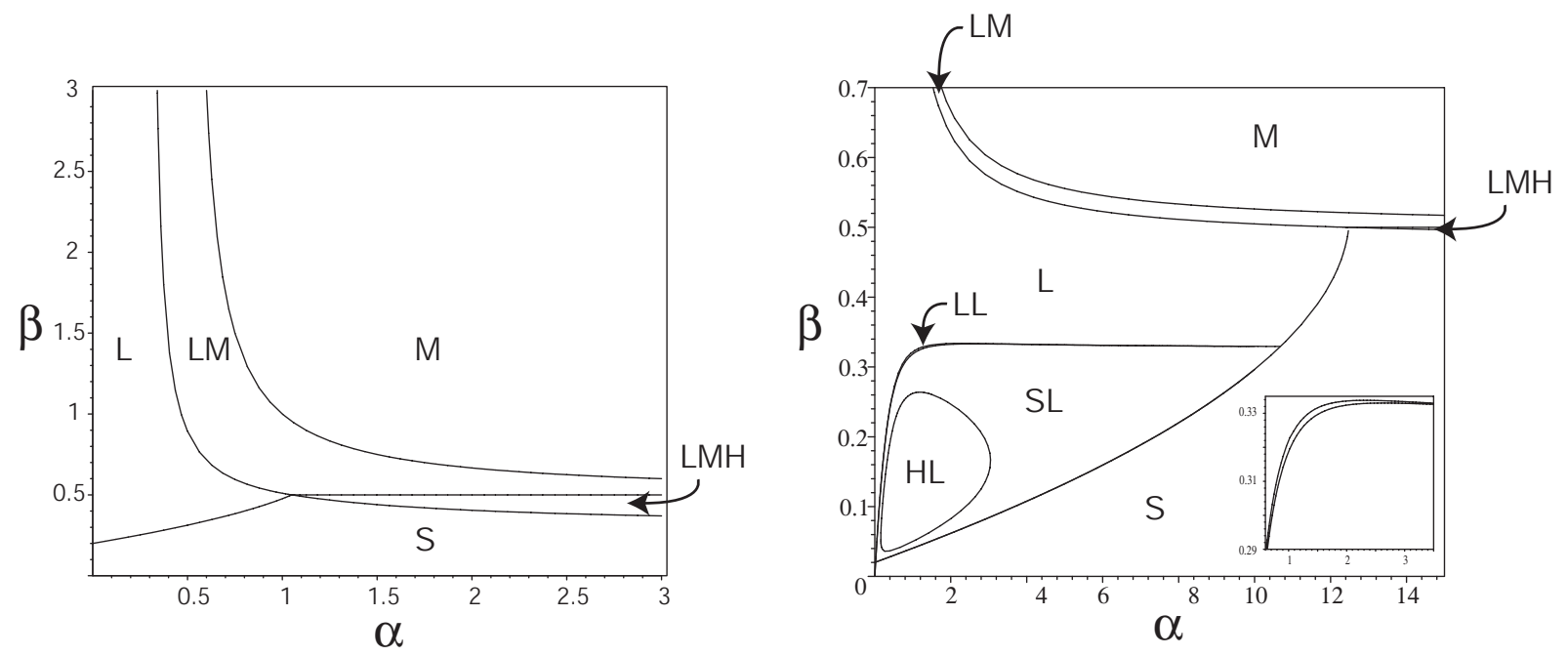

Figure 3. Mean-field phase diagram for the bulk-decoupled case with $\omega_{D}=0$ and $\Omega=0.2$ (left), $\Omega=0.02$ (right). The inset focuses on the regime where the low-asymmetric phase is most pronounced. Abbreviations used for the phases: M maximal-current symmetric phase; LM - Low-Max symmetric phase; LMH - Low-MaxHigh symmetric phase; S - Shock symmetric phase; L - Low symmetric phase; SL Shock-Low asymmetric phase; HL - High-Low asymmetric phase; LL - Low asymmetric phase.

In figure 3 we plot the phase diagram for the cases $\Omega=0.2,0.02$. Note that at the higher value of $\Omega$ no asymmetric phases exist, while at the smaller value all phases described in the previous section exist. This fact will be addressed below. Taking $\Omega$ to zero the original phase diagram of the Bridge model is retrieved, as discussed in subsection 3.2 .3 .

3.2.1. Stability. As mentioned above, all asymmetric phases reside in regions of phase space in which the symmetric low density is also a stationary solution of the mean-field equations. On the mean field level, the realization of one stationary solution rather than the other is a matter of initial conditions. In all cases both the symmetric and asymmetric solutions are linearly stable. However, any initial condition for which the density of at least one of the two species is higher than $\frac{1}{2}$ in some region evolves into the state of broken symmetry. Thus a disordered initial condition, in which the density of particles at any site is an independent uniformly distributed random variable, resides in the basin of attraction of the asymmetric solution.

Considering the model beyond mean-field approximation, where the dynamics is noisy, one expects a random perturbation to take the system away from the symmetric solution. In physical terms this can be understood by the fact that nucleation of a high density domain leads to its flow towards the boundary, where it reduces the inflow of particles of the other species due to the exclusion interaction. Once the symmetry is broken, the high density phase expands and takes its steady state position. This picture is substantiated in a quantitative manner for the limit $\beta \rightarrow 0$ in section [6] Note that 
this line of argument cannot be followed for the asymmetric Low phase.

3.2.2. Extinction of the asymmetric phases at high $\Omega$. So far we have considered the case $\omega_{D}=0$, where detachment of particles from the bulk is suppressed. In this case the non-conserving rates allow for attachment of particles of either species with rate $\omega_{A}$ and for charge exchange with rate $\omega_{X}$. While the former process affects the densities of both species in the same way, the charge exchange process tends to diminish the density difference between species. Thus it is clear that for large $\omega_{X} \sim \Omega$, when this process becomes dominant, the asymmetric phases will vanish. This effect can only increase in the presence of detachment, which acts stronger on the majority species. In the case $\omega_{D}=0$ we find that the High-Low phase ceases to exist beyond $\Omega \simeq 0.035$. The Shock-Low phase vanishes at $\Omega \simeq 0.138$, and with it the Low asymmetric phase. The vanishing of the asymmetric phases can be understood in a more quantitative manner from the blockage picture described in section 7

3.2.3. The limit $\Omega \rightarrow 0$. In the limit of $\Omega \rightarrow 0$ the non-conserving model considered here must coincide with the Bridge model. In this limit, the point $R$ defined above is shifted towards the origin. The point $Q$ is pushed to infinity, which means that the Low-Max-High symmetric phase cannot exist. The boundary line between the Low symmetric and the shock symmetric phase is given generally by

$$
\beta_{\mathrm{LS}}=\Omega+\frac{1}{2}-\frac{1}{2} \sqrt{1-4 \alpha \Omega} \text { for } \alpha \leq \frac{1-4 \Omega^{2}}{4 \Omega} .
$$

Thus $\beta_{\mathrm{LS}} \rightarrow 0$ for all $\alpha$ as $\Omega \rightarrow 0$, and the shock symmetric phase cannot exist. Furthermore the Low-Max symmetric phase vanishes in this limit. The boundary between this phase and the Maximal-current phase,

$$
\beta=\frac{\alpha}{2 \alpha-1} \text { for } \alpha>\frac{1}{2},
$$

coincides in the limit $\Omega \rightarrow 0$ with its boundary with the Low phase,

$$
\beta=\frac{\alpha}{2 \alpha-1+4 \Omega(\alpha+\Omega)} \text { for } \frac{1}{2}<\alpha<\frac{1-4 \Omega^{2}}{4 \Omega} .
$$

Thus, of the symmetric phases only the Maximal-current phase and the Low phase remain in the $\Omega \rightarrow 0$ limit, as expected from the Bridge model.

As for the asymmetric phases, one notices in the same way that as $\Omega$ decreases, the High-Low phase region grows on the expense of the Shock-Low phase. As $\Omega \rightarrow 0$, the boundary lines of both phases coincide, and the Shock-Low phase ceases to exist. Exact expressions for these phase boundaries are rather lengthy, and are omitted here. Finally, the low density asymmetric phase takes in the $\Omega \rightarrow 0$ limit its form as in the Bridge model. 


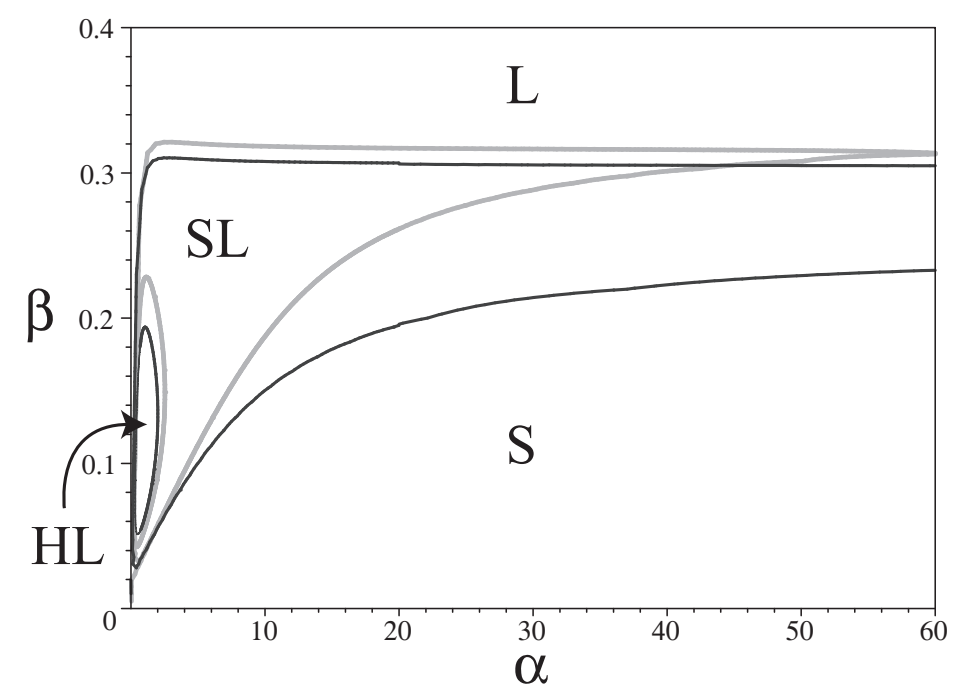

Figure 4. Mean-field phase diagram for the bulk-decoupled case, with $\Omega=0.02$ and $u_{D}=1$ (gray lines), $u_{D}=2$ (dark lines). The boundary line between the asymmetric low-density phase and the symmetric Low phase is omitted. Abbreviations for the different phases are the same as in figure 3

\subsection{Detachment from the bulk: the case $\omega_{D} \neq 0$}

We now turn to consider a more general case, still within the bulk-decoupled regime $\left(q=1, \omega_{D}=\omega_{X}\right)$, where the non-conserving dynamics includes detachment of particles. This case corresponds to the NC-TASEP with non-equal attachment and detachment rates. The phase diagram of that model includes only three phases: high density, low density and localized shock. All phases where a part of the profile is constant at $\frac{1}{2}$ do not exist. This comes from the fact that the equilibrium density of the kinetics is given by the Langmuir density rather than $\frac{1}{2}$.

The phase diagram of our model in the case $\omega_{D} \neq 0$ exhibits only two symmetric phases - the symmetric low-density phase and the shock symmetric one. All asymmetric phases which were obtained for the case $\omega_{D}=0$ are also present here. As in the NCTASEP, the density profiles in this case are not linear, but rather curved. This makes the analysis of the phase diagram somewhat more cumbersome, although not different in principle from the one presented in section 3.1. Details of this calculation are given in the appendix.

In figure 4 we plot the mean-field phase diagram for the cases $u_{D}=1$ and $u_{D}=2$, where $u_{D}$ is defined as before by $u_{D}=\omega_{D} / \omega_{A}$. The boundary line between the asymmetric low-density phase and the symmetric Low phase is not presented. We could not obtain the boundary densities in this phase in a closed form. For several values of $u_{D}$ we have found numerically that this line lies just above the transition line between the Shock-Low phase and the asymmetric Low phase. Certainly, the region of phase space covered by this phase does not increase compared with the case $\omega_{D}=0$. It is readily noticed that the part of phase space spanned by the High-Low asymmetric 
phase decreases as $\omega_{D}$ is increased. This is expected from the fact that the detachment process acts stronger on the majority phase, thus reducing its density. For any given $\Omega$ the detachment process can be increased to a level in which the High-Low asymmetric phase does not occur.

The detachment process can be considered as cooperating with the boundary ejection rate $\beta$, and competing with the boundary injection rate $\alpha$. It is no surprise then that the asymmetric Shock-Low phase grows in the $\alpha$ direction of phase space and shrinks in the $\beta$ direction as $\omega_{D}$ is increased. In the regions of phase space which compose the three phases identified only for the case $\omega_{D}=0$, the density profiles approach continuously, as $\omega_{D}$ is decreased towards zero, to the ones described in the corresponding phases of the $\omega_{D}=0$ case.

\section{Monte-Carlo simulations}

As was already noticed in earlier works about the Bridge model [6, 9, the mean field phase diagram captures the correct topology of the phase boundary lines. The exact location of the boundary lines, however, is shifted in the noisy model with regard to those of the mean field solution. For our model we did not try to obtain the exact location of the phase boundary lines from Monte-Carlo (MC) simulations. Still we note, based on our simulations, that these lines cannot lie too far from those of the mean-field phase diagram obtained in the previous section. Here we concentrate on giving evidence for each of the phases by finding representative points in parameter space. In figure 5 we present the density profiles of the two species in the different phases as obtained from $\mathrm{MC}$ simulations. The profiles in each phase were taken at the same parameters as the respective mean field profiles shown in figures 1 and 2. For all phases shown here the mean field profiles capture the features of the noisy model. While $\mathrm{MC}$ simulations were done for a system of size $N=2000$, the mean field results are obtained for $N=128$. A quantitative comparison between the profiles becomes meaningful in the limit $N \rightarrow \infty$ using rescaled coordinates $x=i / N$. In this limit the localized shocks in the Shock-Low and symmetric Shock phase become sharp [11]. In figure 5 the density profile for the Low asymmetric Phase is omitted. This phase is addressed in the following subsection.

\subsection{The Low asymmetric phase}

The existence of the Low asymmetric phase is an issue of longstanding discussion [9, 8, It was noted already in the mean-field solution of the Bridge model [6] that the region in phase space covered by this phase is very small compared to the others. Furthermore MC simulations indicate that the particle densities in this region of phase space fluctuate strongly [8]. These facts also hold true in the model considered here. Therefore we refrain from presenting a $\mathrm{MC}$ density profile as was done for the other phases. In [8] the

existence of the Low-asymmetric phase in the Bridge model is deduced from sampling 

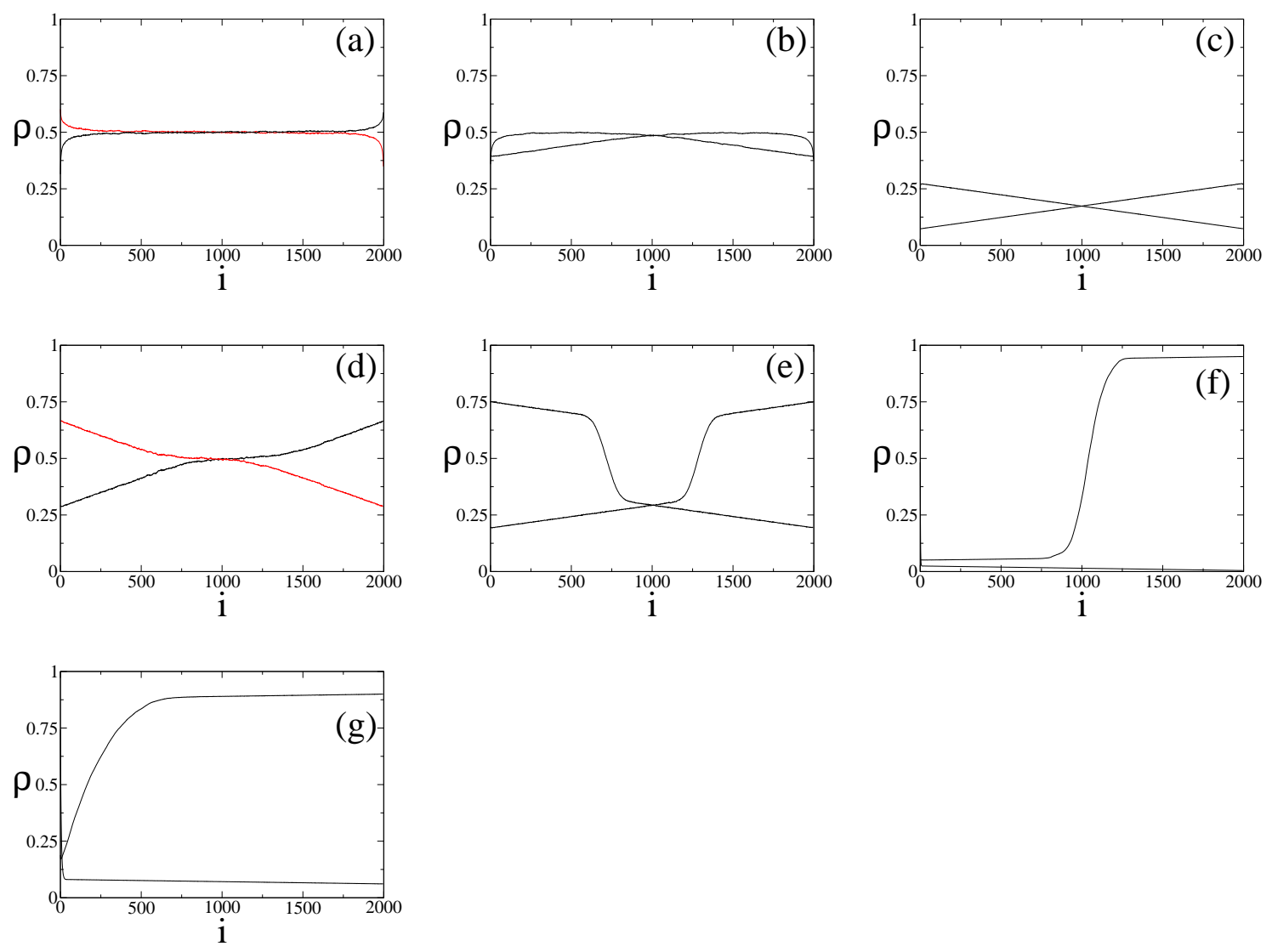

Figure 5. Density profiles as obtained from a Monte-Carlo simulation of a system of size $N=2000$. (a) Max Phase, $\alpha=3.0, \beta=0.8, \Omega=0.2, q=1$. (b) Low-Max Phase, $\alpha=1.0, \beta=0.7, \Omega=0.2, q=1$. (c) Low Phase, $\alpha=0.1, \beta=0.8, \Omega=0.2, q=1$. (d) Low-Max-High Phase, $\alpha=5.0, \beta=1 / 3, \Omega=0.5, q=1$. (e) Shock Phase, $\alpha=3.0$, $\beta=0.25, \Omega=0.2, q=1$. (f) Shock-Low asymmetric Phase, $\alpha=0.1, \beta=0.05$, $\Omega=0.02, q=1$. (g) High-Low asymmetric Phase, $\alpha=1.5, \beta=0.1, \Omega=0.02, q=1$.

the probability distribution $P(\bar{p}, \bar{m})$, where $\bar{p}$ and $\bar{m}$ are the average densities of positive and negative particles in the system respectively. In our model, this line of argument fails. On the level of average densities, the Low-Low and Shock-Low phases cannot be distinguished. This is because both phases can exhibit distributions $P(\bar{p}, \bar{m})$ with two peaks at $\bar{p}$ and $\bar{m}$ smaller than $\frac{1}{2}$.

The blockage picture outlined in section 7 yields no arguments in favor of the Low asymmetric phase. At the upper boundary of the Shock-Low phase the localized shock position retracts to $x_{s}=0$. This allows particles of both species to enter the system. A symmetry broken Low phase beyond this point would require some kind of blockage being formed at the exit of the majority species. The nature of such a blockage is not clear. It remains to be clarified, whether the existence of this phase, which is evident in the mean field treatment, can be demonstrated in the noisy model. 

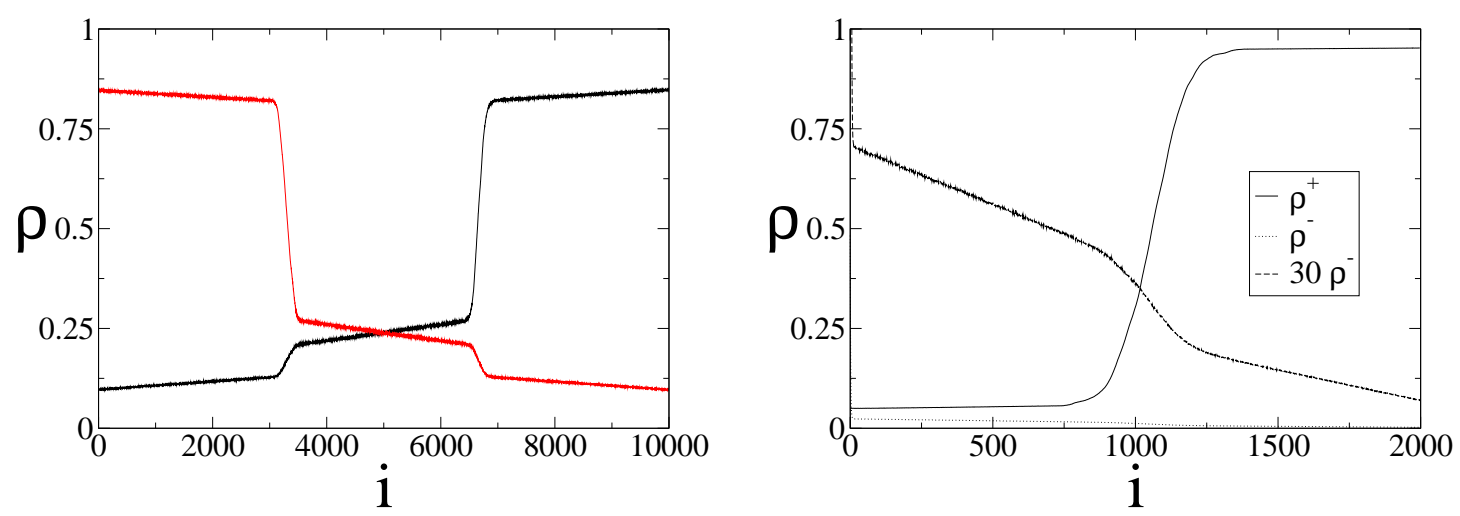

Figure 6. Induced shocks. (Left) Density profiles in the symmetric shock phase, as obtained in Monte-Carlo simulations. A primary shock in one of the species is accompanied by an induced shock in the other species. Here $q=2$ and $\alpha=3.0$, $\beta=0.25, \Omega=0.02$. (Right) Density profiles in the Shock-Low phase, as obtained in Monte-Carlo simulations. The localized shock in the majority species is accompanied by an induced shock in the minority species. The profile of the minority species is additionally shown when multiplied by a factor of 30 , in order to demonstrate the induced shock phenomenon. Here $q=2$ and $\alpha=0.1, \beta=0.05, \Omega=0.02$.

\section{Induced shocks}

In the case $q \neq 1$ the bulk dynamics of the two particle species are not decoupled. Thus, one cannot solve the mean field equations in the way it was done in section [3. Still, the phase structure can be explored by integrating the mean field equations numerically, or by $\mathrm{MC}$ simulations. It shall not be attempted here to give the full phase diagram of the model. We do note, however, that phases with broken symmetry exist also in the general case.

In the Shock symmetric phase and the Shock-Low phase the coupling of the dynamics of the two species gives rise to an induced shock phenomenon. Here the existence of a localized shock in the density profile of one species induces a shock in the density profile of the other species. For example, in the Shock symmetric phase one notices that the density profile of each one of the species exhibits actually two shocks in the steady state (figure 6). One is a primary shock, created by the same localization mechanism which was already identified in the bulk-decoupled case. The existence of this shock, albeit not its detailed properties, relies only on the properties of the density profile of the very same species. The second shock is induced by the primary shock in the density of the other species, and it shares its location. Across both shocks the current is continuous. This phenomenon also occurs in the Shock-Low phase (figure Gi). Here, the localized shock in the majority phase induces a shock in the Low phase. In fact, it was shown in [14] that for general two species systems with coupled density-current relation a shock in one particle species induces a shock in the other one.

For general $q$ the current-density relation of, say, the positive particle species, 
$j_{i}^{+}\left(p_{i}, m_{i}\right)$, depends on the local density of both species. In general this current-density relation is not known, except for two cases: the case $q=1$ (the decoupled case), where $j^{+}=p(1-p)$, and the case $q=2$, where $j^{+}=p(1-p+m)[$ ] .

As discussed in 13 the current across a localized shock is continuous. This requirement implies when $q=1$ that shocks are symmetric with respect to $p=1 / 2$, irrespective of the local density $m$ of particles of the opposite species. In general, however, this is not the case. The properties of both the primary and induced shock in the density profile of each species rely on the local densities of both.

The continuity of the current across the shocks can in principle be used to determine the properties of the primary and induced shocks, if one can develop the density characteristics from the boundaries of the system. For the case $q=1$ the equations for the two density profiles are decoupled, and one uses this method to determine the position of the shock. Of course, no induced shocks are present in this case. For general $q$, however, the equations for the density profiles are coupled, and an analytical solution is not available.

\section{Exact solution for the limit $\beta, \Omega \rightarrow 0$}

In [7] a toy model was presented to allow for the exact solution of the limit $\beta \rightarrow 0$ of the Bridge model. In this section a generalization of the toy model is presented. The solution of this model gives an exact description of the $\beta \rightarrow 0, \Omega \rightarrow 0$ limit of the model, and proves that to lowest order in $\beta$ mean-field theory recovers the exact phase diagram.

In the limit $\beta \rightarrow 0, \Omega \rightarrow 0$ the only relevant configurations are those composed of three blocks, containing (from left to right) negative particles, vacancies, and positive particles. A configuration of this type is long-lived, as all exit rates from it scale to zero. In this limit, all other configurations rearrange themselves into one of these three-block configurations. A configuration of this type is identified by two variables, $y$ and $x$, defined as the size of the left (negative particle) block and the right (positive particle) one, respectively. Thus, for example,

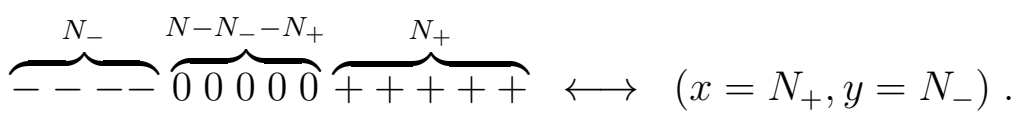

Let us assume that the system is in a three-block configuration $(x, y)$, and consider the ways it can leave it. First, a particle can leave the system through a boundary with rate $\beta$, leaving a vacancy behind it. This vacancy can travel into the system with rate $(1+\alpha)^{-1}$, in which case the system is again in a three-block configuration. Otherwise, the particle which had left the system can be replaced by a particle of the opposite species with rate $\alpha(1+\alpha)^{-1}$. On a short time scale this particle travels through the system until it joins the block on the other side, thus returning the system into a three-block state.

Another possible way out of a three-block configuration is through the nonconserving processes in the bulk. First, a particle can be attached to the system in the vacancy domain with rate $\omega_{A}(N-x-y)$. This particle joins on a short time scale 

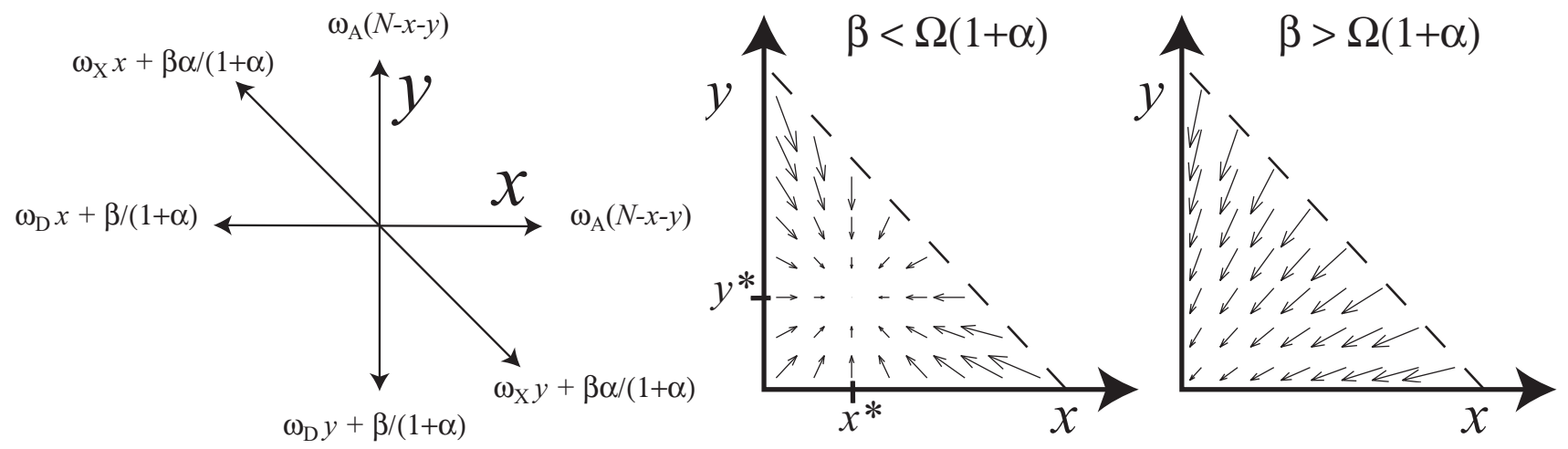

Figure 7. Toy model for the limit $\beta, \Omega \rightarrow 0$. (Left) The rates defining the corresponding random-walk model (38). (Right) Flow fields of the model.

to the block of its own species. Second, a positive (negative) particle can be detached from the system with rate $\omega_{D} x\left(\omega_{D} y\right)$, thus creating a vacancy within a particle block. On a short time scale this vacancy travels into the system and joins the vacancy block. Finally, a positive (negative) particle can change its species with rate $\omega_{X} x\left(\omega_{X} y\right)$, and move from one particle block to the other.

When the last particle of its species leaves the system, the other type of particles can rush into the system through the boundary. The system fills rapidly with particles of this type. Thus, the only possible configurations with $x=0$ or $y=0$ are $(0, N)$ and $(N, 0)$.

To summarize, consider a two-dimensional random walker, whose position $(x, y)$ corresponds to the block-configuration of the two-species system. The transition rates for this walker are (see also figure 7)

$$
\begin{array}{ll}
(x, y) \rightarrow(x+1, y) & \text { with rate } \omega_{A}(N-x-y) \\
(x, y) \rightarrow(x, y+1) & \text { with rate } \omega_{A}(N-x-y) \\
(x, y) \rightarrow(x-1, y) & \text { with rate } \beta \frac{1}{1+\alpha}+\omega_{D} x \\
(x, y) \rightarrow(x, y-1) & \text { with rate } \beta \frac{1}{1+\alpha}+\omega_{D} y \\
(x, y) \rightarrow(x-1, y+1) & \text { with rate } \beta \frac{\alpha}{1+\alpha}+\omega_{D} x \\
(x, y) \rightarrow(x+1, y-1) & \text { with rate } \beta \frac{\alpha}{1+\alpha}+\omega_{D} y \\
(1, y) \rightarrow(0, N) & \text { with rate } \beta \\
(x, 1) \rightarrow(N, 0) & \text { with rate } \beta .
\end{array}
$$

It can be shown that this toy model is mapped exactly to the two-species model in the limit $\beta, \Omega \rightarrow 0$, in the sense formulated in appendix $\mathrm{A}$ of [7].

Let us first consider the case where the dynamics defined in (38) leads to a fixed point solution. This is the case where the net flows on both the $x$ and $y$ directions 
vanish at some point $\left(x^{*}, y^{*}\right)$. The fixed points must satisfy the equations

$$
\begin{aligned}
& \left(\omega_{X}-\omega_{A}\right) x^{*}-\left(\omega_{X}+\omega_{A}+\omega_{D}\right) y^{*}=-\omega_{A} N+\frac{\beta}{1-\alpha} \\
& -\left(\omega_{X}+\omega_{A}+\omega_{D}\right) x^{*}+\left(\omega_{X}-\omega_{A}\right) y^{*}=-\omega_{A} N+\frac{\beta}{1-\alpha},
\end{aligned}
$$

whose solution is

$$
\frac{x^{*}}{N}=\frac{y^{*}}{N}=\frac{1}{2+u_{D}}\left(1-\frac{\beta}{\Omega(1+\alpha)}\right) .
$$

Interestingly, the fixed point does not depend on the charge exchange rate $\omega_{X}$. Notice that this fixed point can only exist if $0 \leq x^{*} / N, y^{*} / N \leq 1$. Indeed, $x^{*}$ and $y^{*}$ of (40) always meet the second condition. The first condition, however, is only met for

$$
\beta<\Omega(1+\alpha) .
$$

Otherwise, the random walker is always biased towards the axis of the $(x, y)$ plane which is closer to its position (see figure [7). In this case, $\Omega<\beta \rightarrow 0$, one recovers the toy model of 7 which yields a stable state of broken symmetry. One of the species then occupies most of the lattice, corresponding to the High-Low asymmetric phase of the model.

Thus, the toy model yields two phases in the limit $\beta, \Omega \rightarrow 0$. For $\beta<\Omega(1+\alpha)$ one has a symmetric phase, with a dominating three-block configuration described by (40). This corresponds to a symmetric shock phase in the model, where the shock position of the positive particles is $x_{s}=1-x^{*} / N$, with $x^{*}$ given by (40). Otherwise the system is in the High-Low asymmetric phase, with the line $\beta=\Omega(1+\alpha)$ serving as the transition line between the two phases.

For the bulk-decoupled case, $\omega_{A}=\omega_{X}$ and $q=1$, it is illuminating to compare these exact results with the ones obtained by mean field. The mean field analysis, performed in section 3, predict in the limit $\beta, \Omega \rightarrow 0$ the two phases obtained in the toy model. The mean field transition line between the two phases (23) is identical, to first order in $\beta$, to the line $\beta=\Omega(1+\alpha)$ of the toy model. Also the shock position $x_{s}$ calculated in the toy model is identical to first order in $\beta$ to the one (24) calculated in mean field. This result also holds in the case $\omega_{D} \neq 0$. We thus conclude that the mean-field solution is exact to first order in $\beta$.

\section{Blockage picture}

In this section we combine the mean-field and stability analysis, the simulation results, and the toy model into a physical picture. Following [8] we term it the blockage picture.

Qualitatively, typical configurations in the asymmetric phases can be described in terms of blocks of the two species, which spread from the 'exit' boundary into the system (figure 8 , and compare the toy model description in the previous section). The density profile within each block is not constant, but this feature is not relevant here. As mentioned above, a block of one species stalls the entry of particles of the other 

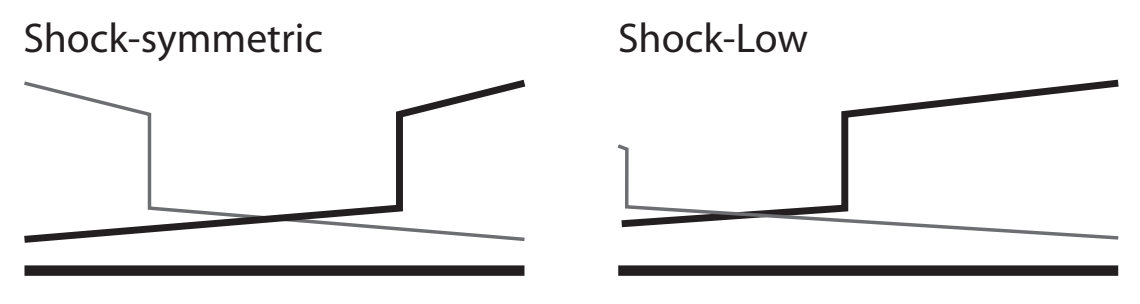

High-Low

Low-asymmetric
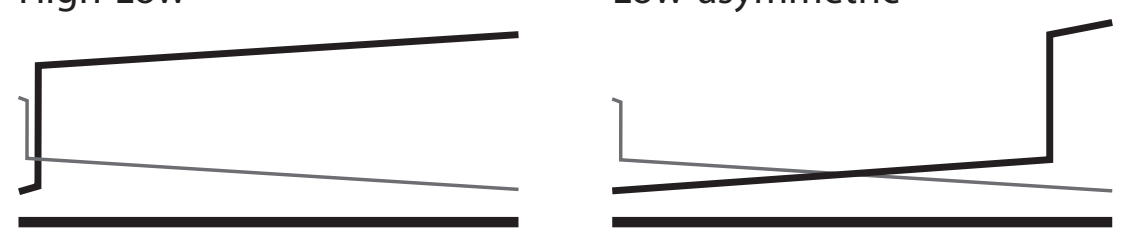

Figure 8. Blockage picture. Schematic picture of the instantaneous density profiles. Density profiles of positive particles are depicted by dark lines, negative particles by gray lines. Here, as in the text, we assume that the positive particles are the majority species.

species through the boundaries, thus serving as a blockage. The possibility of particles to enter the system in the bulk serves to stabilize the domain size.

In the High-Low phase the block of the majority species covers the entire system, while in the Shock-Low phase the block is limited to some part of the system. The fluctuations in the size of this block, corresponding to the width of the localized shock, are limited to an area of size $\sim N^{-1 / 2}$ [11]. The minority block in both phases is unstable in the sense that the domain wall between it and the bulk region drifts towards the boundary. Averaging over the positions of the domain wall results in the exponential decay of the mean field density profile from the left boundary.

In [8] it was observed that an instantaneous configuration in the low-density asymmetric phases comprises a small block of the majority phase, which is limited to the vicinity of the boundary. The formation of this block prevents particles of the other species from entering the system, thus leading to symmetry breaking. However, this block does not survive for times which are exponential in the system size.

The block picture is extended into the symmetric shock phase. Here the two blocks are covering equal distances from the 'exit' boundaries. The sizes of the two blocks are again macroscopic and localized, in the sense that the size fluctuations vanish as $N^{-1 / 2}$.

We now turn to describe the different phases of the model in the language of block configurations. To this end we take a stroll along a line of constant $\alpha$ in the phase diagram, starting from the symmetric shock phase and going up in $\beta$. This line is chosen such that it cuts through all asymmetric phases.

In the shock symmetric phase, the two blocks inhibit, in a symmetric way, the inflow of particles of the opposite species. Increasing $\beta$ decreases the sizes of the two blocks. However, as long as their size is macroscopic, the blocks keep their role of lessening the ability of particles of the other species to enter the system. 

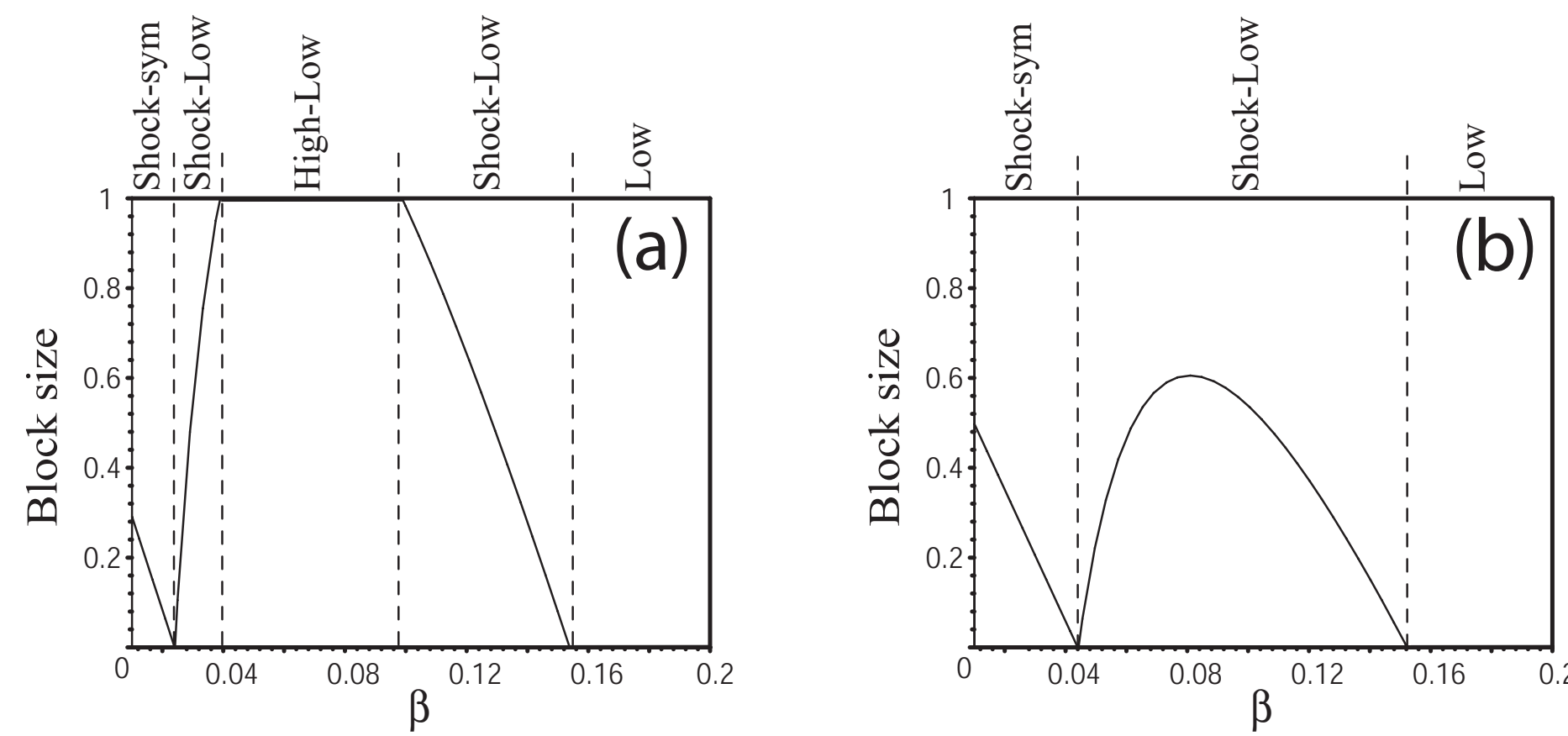

Figure 9. Blockage size as a function of $\beta$, as calculated in mean-field for the bulkdecoupled case. Here $\alpha=0.2, \omega_{D}=0$ and (a) $\Omega=0.02$, (b) $\Omega=0.03$.

As the boundary line is approached, the size of the blocks is reduced to zero. Now the road is open for both species to enter the system. Due to fluctuations, the formation of a block is inevitable. As $\beta$ is increased beyond the transition line into the Shock-Low phase, the possibility rises that this block will be stabilized by the nonconserving dynamics. A spontaneously created block of one species, which now has a stable macroscopic size, hinders particles of the other species and breaks the symmetry between the two species.

As $\beta$ is increased from its value at the boundary line between the symmetric shock phase and the Shock-Low phase, the size of the block of the majority species increases. This is due to the coupling between the ejection rate $\beta$ and the effective injection rates, which at this region of phase space serves to increase $\alpha^{+}$(in mean-field this can be seen from Eqs. 13, 251). At some value of $\beta$, the block reaches the size of the entire system, and there it stays for some range of $\beta$. This, in fact, is the High-Low phase of the system. As $\beta$ is increased further the size of the majority block shrinks back, and the system is again in the Shock-Low phase. The transition from the Shock-Low phase to the High-Low phase at some $\beta$, and the re-entrance to the Shock-Low phase at some higher $\beta$, occur at these points where the size of the majority blockage becomes identical with the size of the system.

Towards the boundary line between the Shock-Low phase and the low-density phase the size of the majority block vanishes. The existence of a reminiscent block which yields the asymmetric Low phase, as discussed in [8], can be either attributed to fluctuations of the localized shock, or to an alternative mechanism. 
To make the blockage picture more quantitative, we calculate the size of the majority block in each phase. This is done within mean-field for the bulk-decoupled case. For simplicity we take $\omega_{D}=0$, where the profiles of the blocks are linear. The block size is then just $1-x_{s}$, where the shock position $x_{s}$ is given in (24). In figure 9 (a) we plot the size of the block as a function of $\beta$ at constant $\Omega$ and $\alpha$. Using the picture described above, one can identify the phase boundaries. The value of $\beta$ at which the blockage first disappears corresponds to the Shock-symmetric to Shock-Low line. The two values between which the blockage spans the system are identified as the two lines between the Shock-Low and High-Low phases. Finally, the higher $\beta$ at which the blockage disappears completely corresponds to a transition into a low-density phase. It is easy to verify, by comparing with the mean-field phase diagram, that these are indeed that transition points between the phases. Note that the Low asymmetric phase escapes this picture.

It is also possible to describe in terms of the block size (or alternatively the shock position) the fact that asymmetric phases disappear as $\Omega$ is increased. The nonconserving dynamics in the bulk of the system serves to sustain the localized shock. Keeping $\alpha$ constant, for example, the increase in the amplitude of the non-conserving rates drives the position of the shock out of the system, thus decreasing the maximal size of the blockage. In terms of phases, this would decrease the segment on the $\beta$ axis in which the shock is localized at the 'entry' boundary (i.e. the High-Low phase), down to a point where the shock cannot get so far and the phase disappears. Beyond this point, as depicted in figure 9 (b), the position of the shock is driven back towards the 'exit' boundary, thus reducing the Shock-Low phase until it is finally gone.

\section{Summary}

In this work a two-species one-dimensional model, with dynamics which is not conserving both at the boundaries and in the bulk, has been studied. The dynamics is symmetric under charge exchange and left-right reflection. By definition the non-conserving dynamics in the bulk of the system acts to diminish the difference between the densities of the two species. Nevertheless we have found that the symmetry between the two species can be broken even in the presence of bulk non-conserving processes.

The mean-field phase diagram, obtained for the case where the bulk dynamics of the two species becomes decoupled, exhibits three phases in which the symmetry between the species is broken. One of these is unique to the case where the bulk dynamics is not conserving, and results from a localization of shocks in the density profiles. All asymmetric phases reside in regions of phase space where symmetric low-density profiles are another fixed point of the mean-field dynamics. However, stability arguments shows that it is the asymmetric solution which should be expected to survive fluctuations. Indeed, comparing with Monte-Carlo simulations, two asymmetric phases are confirmed. The third, in which the average density of both species is below $\frac{1}{2}$ is more difficult to determine. 
In contrast to the bulk-conserving case, in this model the density profiles are generally not flat. In particular, localized shocks may be generated in the bulk of the system. In the general case, when the particle current of one species depends on the density of the other, a localized shock in the density profile of one species induces a shock in the other.

In the asymmetric phases, as well as in the shock symmetric phase, typical configurations can be described in terms three blocks. The leftmost block has a high density of negative particles, the middle block has a low density of particles of both species, and the right block is mainly occupied by positive particles. This observation serves to define a toy model which describes the dynamics of the system in terms of a two-dimensional random walker. Solving the toy-model yields an exact solution for the case where the exit rates and the non-conserving rates are taken to zero. The results coincide with the ones obtained in mean-field at this limit. For the general case a more qualitative picture emerges, which serves to describe the phase transitions in the model in terms of the block sizes.

The bulk of this work, as well as of those which studied the Bridge model, has focused on the case in which the dynamics of the two species is decoupled in the bulk. The other, more general case, was studied only by numerical means, both on the meanfield level and in Monte-Carlo simulations. This enabled us to observe induced shocks. A more detailed study of this case by analytical means should shed more light on this phenomenon.

The mean-field phase diagram of the NC-TASEP is expected to be exact [13, while Monte-Carlo results suggest that this is not the case here. Such is also the case in the Bridge model. It should be interesting to study the correlations which build up in the system, taking it away from the mean-field description.

\section{Acknowledgments}

It is a pleasure to thank D. Mukamel, and G.M. Schütz for suggestions, discussions and critical reading of the manuscript. We thank M.R. Evans, and M. Paessens, and V. Rittenberg for useful discussions. The Einstein Center and the Forschungszentrum Jülich are gratefully acknowledged for support during mutual visits. The support of the Israeli Science Foundation is gratefully acknowledged.

\section{Appendix A. Mean-Field analysis of the case $\omega_{D}>0$}

In this Appendix we discuss the construction of the phase diagram for the bulk-decoupled case $q=1, \omega_{A}=\omega_{X}$ with $\omega_{D} \neq 0$. This case corresponds to the NC-TASEP with nonequal attachment and detachment rates (the case $K \neq 1$ in [11, 13, 12]). The phase diagram of the model in the case $\omega_{D} \neq 0$ exhibits two symmetric phases, symmetric low-density phase and shock symmetric one, and three asymmetric ones, High-Low density phase, Shock-Low phase, and low-density asymmetric phase. The profiles do 
not have constant slopes as in the $\omega_{D}=0$ case. Starting from left boundary density $\alpha$, the density at the right boundary resulting from the left characteristic is given by

$$
\rho_{N}^{(\ell)}(\alpha)=\frac{2-u W_{0}\left(\frac{(2-4 \alpha-2 \alpha u)}{u} \exp \left(-\frac{4 \alpha+2 \alpha u+\Omega(2+u)^{2}-2}{u}\right)\right)}{2(2+u)} .
$$

The respective expression for the density at the left boundary resulting from the right characteristics starting from density $1-\beta$ reads

$$
\rho_{1}^{(r)}(\beta)=\frac{2-u W_{-1}\left(\frac{(4 \beta-2-2 u+2 \beta u)}{u} \exp \left(\frac{\left.\Omega(2+u)^{2}-2(1+u)+2 \beta(2+u)\right)}{u}\right)\right)}{2(2+u)} .
$$

Our aim is to use the known phase diagram of the NC-TASEP [11] to construct the phase diagram of the two-species model, as it was done in section 3.1. To this end, let us define the two transition lines in this phase diagram. The first is the transition line between the high density phase and the localized shock phase,

$$
\beta_{\mathrm{HS}}(\alpha)=\frac{2(1+u)+u \mathrm{~W}_{0}\left(\frac{(4 \alpha-2-2 u+2 \alpha u)}{u} \exp \left(\frac{-4 \alpha+2+2 u-2 \alpha u+\Omega(2+u)^{2}}{u}\right)\right)}{2(2+u)} .
$$

where $\mathrm{W}_{k}(z)$ is the Lambert-W function. This equation defines the line only for $\alpha<1 / 2$. The second transition line separates the shock phase and the low density phase. This line is given, for $\alpha<1 / 2$, by

$$
\beta_{\mathrm{SL}}(\alpha)=\frac{2-u \mathrm{~W}_{-1}\left(\frac{(2-4 \alpha-2 \alpha u)}{u} \exp \left(-\frac{4 \alpha+2 \alpha u+\Omega(2+u)^{2}-2}{u}\right)\right)}{2(2+u)} .
$$

Both boundary lines are continued for $\alpha>1 / 2$ by $\beta_{\mathrm{HS}}\left(\frac{1}{2}\right)$ and $\beta_{\mathrm{SL}}\left(\frac{1}{2}\right)$, respectively.

Let us recall the procedure in which one obtains the phase diagram for the twospecies model. For each phase, one obtains from (13) the effective boundary rates, $\alpha^{+}$ and $\alpha^{-}$. This requires knowledge of the four boundary currents, $j_{b}^{+}=p_{b}\left(1-p_{b}\right)$ and $j_{b}^{-}=m_{b}\left(1-m_{b}\right)$, where $b=0, N$ for the left and right boundaries. The boundary lines are then obtained from comparing the effective boundary rates with the corresponding transition lines of the NC-TASEP.

We do not repeat the analysis here in such details as it was done for the case $\omega_{D}=0$. Instead, we give for each phase the four boundary densities, needed to calculate the boundary currents and the effective rates. In addition the conditions on the effective rates, which define the phase boundaries, are given in terms of $\beta_{\mathrm{HS}}$ and $\beta_{\mathrm{SL}}$ of Eqs. (A.3) and (A.4). The asymmetric low-density phase is omitted, as we could not obtain the boundary densities in this phase in a closed form. The boundary lines for this case were obtained numerically.

Low density symmetric phase. Here, $p_{0}=m_{N}=\alpha^{+}, p_{N}=m_{1}=\rho_{N}^{(\ell)}\left(\alpha^{+}\right)$as in (A.1). The condition for the existence of this phase is

$$
\beta<\beta_{\mathrm{SL}}\left(\alpha^{+}\right)
$$


Shock symmetric phase. The boundary densities are given by $p_{0}=m_{N}=\alpha^{+}$, $p_{N}=m_{1}=1-\beta$. The conditions for this phases existence are

$$
\beta_{\mathrm{SL}}\left(\alpha^{+}\right)<\beta<\beta_{\mathrm{HL}}\left(\alpha^{+}\right) .
$$

Shock - Low asymmetric phase. Let the positive particles be in the shock phase. Then $p_{0}=\alpha^{+}, p_{N}=1-\beta$. The negative particles are in the Low phase, where $m_{N}=\alpha^{-}$

and $m_{1}=\rho_{N}^{(\ell)}\left(\alpha^{-}\right)$as given by equation A.1. The phase exists in a region in phase space where

$$
\beta_{\mathrm{SL}}\left(\alpha^{+}\right)<\beta<\beta_{\mathrm{SL}}\left(\alpha^{-}\right)
$$

High - Low asymmetric phase. As before it is assumed that the positive particles are in the majority phase: $p_{0}=\rho_{1}^{(r)}(\beta)$ according to $\mathrm{A} .2$ and $p_{N}=1-\beta$. The negative particles are in the Low phase: $m_{N}=\alpha^{-}$and $m_{1}=\rho_{N}^{(\ell)}\left(\alpha^{-}\right)$. The High-Low phase exists where

$$
\beta_{\mathrm{HL}}\left(\alpha^{+}\right)<\beta<\beta_{\mathrm{SL}}\left(\alpha^{-}\right)
$$

\section{References}

[1] Schmittmann B and Zia RKP Statistical Mechanics of Driven Diffusive Systems, Vol. 17 of Phase Transitions and Critical Phenomena, edited by C. Domb and J. L. Lebowitz (Academic Press, 1995)

[2] Mukamel D in Soft and Fragile Matter: Nonequilibrium Dynamics, Metastability and Flow, edited by M.E. Cates and M.R. Evans, (Institute of Physics Publishing, Bristol, 2000)

[3] Evans MR Braz. J. Phys. 30 (2000) 42.

[4] Schütz GM in Phase Transitions and Critical Phenomena, Vol. 19, edited by C. Domb and J. L. Lebowitz (Academic Press, 2001)

[5] Schütz GM J. Phys. A: Math. Gen. 36 (2003) R339.

[6] Evans MR, Foster DP, Godrèche C, and Mukamel D Phys. Rev. Lett. 74 (1995) 208 ; J. Stat. Phys. 80 (1995) 69.

[7] Godrèche C, Luck JM, Evans MR, Mukamel D, Sandow S, and Speer ER J. Phys. A: Math. Gen. 28 (1995) 6.

[8] Clincy M, Evans MR, and Mukamel D J. Phys. A: Math. Gen. 44 (2001) 9923.

[9] Arndt PF, Heinzel T, and Rittenberg V J. Stat. Phys. 90 (1998) 783.

[10] Evans MR, Kafri Y, Levine E, and Mukamel D J. Phys. A: Math. Gen. 35 (2002) L433.

[11] Parmeggiani A, Franosch T, and Frey E Phys. Rev. Lett. 90 (2003) 811.

[12] Evans MR, Juhász R and Santen L Phys. Rev. E 68 (2003) 026117.

[13] Popkov V, Rakos A, Willmann RD, Kolomeisky AB, and Schütz GM Phys. Rev. E (2003) 67 066117.

[14] Popkov V and Schütz GM J. Stat. Phys. 112 (2003) 523.

[15] Derrida B, Domany E, and Mukamel D, J. Stat. Phys. 69 (1992) 667 ; Derrida B, Hakim V, Evans MR, and Pasquier V J. Phys. A: Math. Gen. 26 (1993) 1493 ; Schütz G and Domany E J. Stat. Phys. 72 (1993) 277. 